\title{
JOURNAL CLUB ESTRATÉGIA DE ENSINO E APRENDIZAGEM PARA DESENVOLVIMENTO DA ENFERMAGEM BASEADA EM EVIDÊNCIA ${ }^{1}$
}

\author{
JOURNAL CLUB TEACHING AND LEARNING STRATEGY FOR \\ EVIDENCE-BASED NURSING DEVELOPMENT
}

\author{
Vanessa Cecília de Azevedo Michelan ${ }^{1}$ \\ Wilza Carla Spiri
}

\begin{abstract}
Resumo: Estudo de revisão integrativa da literatura. As bases de dados foram: Scopus; Pub Med; ProQuest; Web of Science e BVS, as palavras chaves: journal club e enfermagem ou gestão participativa, no período de 2014 a 2018. O critério inclusão foi: incluir as categorias de artigo (original, revisão de literatura, estudo de caso, multimétodo, análise de conteúdo, entre outros); artigos com resumos e textos completos disponíveis para análise no âmbito nacional e internacional. Foram encontrados 97 artigos, 23 atenderam os critérios de inclusão e constituíram a amostra. Apos análise Bibliométrica extraíram-se quatro categorias tais como: Implementação da estratégia de ensino e aprendizagem Journal Club; Construção do método do Journal Club; As vantagens e desafios da implementação do Journal Club e Avaliação da estratégia Journal Club. Trata-se de uma estratégia promissora, porém pouca utilizada no âmbito nacional, conhecida internacionalmente, mas há necessidades de mais evidências sob sua eficácia ao longo do tempo.
\end{abstract}

Palavras-chave: Journal Club; Enfermagem; Gestão Participativa e Liderança.

\begin{abstract}
Integrative literature review study. The databases were: Scopus; Pub Med; ProQuest; Web of Science and VHL, the key words: journal club and nursing or participatory management, from 2014 to 2018. The inclusion criterion was to include article categories (original, literature review, case study, multi-method, content, among others); articles with summaries and full texts available for analysis at national and international level. We found 97 articles, 23 met the inclusion criteria and constituted the sample. After the Bibliometric analysis, four categories were extracted: Implementation of the teaching and learning strategy Journal Club; Construction of the Journal Club method; The advantages and challenges of implementing the Journal Club and Journal Strategy Review. It is a promising strategy, but little used at the national level, known internationally, but there is a need for more evidence under its effectiveness over time.
\end{abstract}

Keywords: Journal Club, Nursing, Participative Management Leadership.

\footnotetext{
${ }^{1}$ Enfermeira. Doutoranda pela Universidade Estadual Paulista Júlio de Mesquita Filho (UNESP). Botucatu-SP, Brasil. E-mail: vcpazevedo@gmail.com

${ }^{2}$ Doutora em Enfermagem pela Escola de Enfermagem da Universidade de São Paulo. Professora Associada da Universidade Estadual Paulista Júlio de Mesquita Filho (UNESP). Botucatu-SP, Brasil. Email: wilza.spiri@unesp.br
} 


\section{Introdução}

A enfermagem ao longo do tempo vem buscando consolidar e assegurar a sua prática por meio da ciência, ou seja, a atualização na área da saúde ocorre simultaneamente com diversidade de informação dos quais estamos envoltos pelo uso de ferramentas tecnológicas para ampliar a visão do saber no que tange a práxis.

Entretanto, os profissionais estão imersos em altas demandas de trabalho interferindo na disponibilidade para praticar o saber. A ciência tem permitindo a adoção de ferramentas para o estreitamento dos avanços científicos e a prática assistencial (DOMENICO; IDE, 2003).

A Prática baseada em evidências (PBE), define-se como "o uso consciente, explicito e judicioso da melhor evidência atual para tomada de decisão sobre o processo de cuidar individual do paciente" (ATALLAH; CASTRO, 1998, p.7). Originou-se no trabalho do epidemiologista britânico Archie Cochrane e o desenvolvimento deu-se ao longo do tempo paralelo ao acesso a informação (GALVÃO; SAWADA; TREVIZAN, 2004).

A PBE possibilita melhorar a qualidade da assistência, pois evolve a definição de uma situação problema, a busca e avaliação crítica das evidências disponíveis, implementação e avaliação dos resultados, bem como a competência clínica do profissional de enfermagem para tomada de decisão sobre a assistência prestada ao paciente (ATALLAH; CASTRO, 1998).

Segundo a literatura, o profissional de enfermagem e também os educadores em ambos os ambientes clínicos e acadêmicos são exigidos para melhorar as habilidades de PBE dos enfermeiros. Portanto, o pleno engajamento dos profissionais de saúde em acessar, interpretar e aplicar as evidências atuais é fundamental para atingir as metas de melhorias globais no atendimento clínico (MOONAN et al., 2016).

O Gerenciamento Baseado em Evidências (GBE) é definido como a identificação, implementação e avaliação de evidências que direcionam o processo decisório gerencial (PFEFFE; SUTTON, 2006).

Neste sentido, a implementação da ferramenta Journal Club na prática da enfermagem, implicará no auxílio do desenvolvimento de suas competências clínicas para as tomadas de decisões e melhorar a assistência ao paciente.

O Jounal Club, de acordo com literatura, tratava-se de uma tradição antiga para o treinamento nas residências médicas, instituídas por Sir Willian Osler em 1875. Nesta 
época objetivavam compartilhar textos e revisar a literatura em grupos, atualmente incluem discussão e revisão da literatura atual e desenvolvimento de habilidades para avaliar a literatura.

O objetivo final de um Journal Club é melhorar o atendimento ao paciente, incorporando evidências em prática (WILSON et al., 2015). Ampliar o saber em bases fundamentadas, avaliando criticamente a literatura atual, é objeto do Journal Club para a promoção das mudanças na prática clínica (LOPES; LICHTENST, 2007).

$\mathrm{Na}$ enfermagem essa ferramenta, Journal Club, tem sido utilizada para promover a excelência do trabalho da enfermagem por meio da promoção da PBE. Os objetivos são: 1) Aprimorar o conhecimento de enfermagem sobre os resultados da pesquisa atual; 2) Promover a aplicação de modelos de pesquisa clínica e de melhores práticas para a enfermagem; 3) Fornecer um meio pelo qual haja resolução dos problemas clínicos.

Assim, a ferramenta tem sido utilizada para a PBE, no entanto, para GBE ainda existe uma lacuna importante, pois embora essa ferramenta tenha sido proposta há muitos anos indaga-se: Como o emprego do Journal Club vem sendo discutido no âmbito científico nacional e internacional e quais as contribuições para a prática em enfermagem?

Objetivo deste estudo foi sintetizar as produções científicas sobre o tema Journal $C l u b$ na área de enfermagem, caracterizando as publicações segundo: identificação do artigo original (título, autores, ano, periódico e país), características metodológicas do estudo, considerações finais e conclusões.

\section{Procedimentos Metodológicos}

Estudo de revisão integrativa, o qual buscou por produções sobre o tema Journal Club, entre o período de 2014 a 2019. A revisão integrativa é uma parte valiosa do processo de criação e organização de um corpo da revisão da literatura; permite construir uma análise ampla e inclusive aborda discussões sobre os métodos e resultados das publicações (GANONG, 1987).

O principal objetivo desta revisão visa fornecer uma síntese dos resultados de pesquisas, identificando as ideias dos especialistas sobre o assunto analisado.

Para a elaboração da presente revisão integrativa adotou-se as seguintes etapas: identificação do tema ou formulação da questão norteadora; amostragem ou busca na literatura dos estudos; categorização dos estudos; avaliação dos estudos incluídos na 
revisão; discussão e interpretação dos resultados e a síntese do conhecimento evidenciado nos artigos analisados ou apresentação dos resultados da revisão integrativa.

O método dessa revisão integrativa seguiu seis etapas (GANONG, 1987, p.8 ):
1) Seleção das hipóteses ou questões para a revisão;
2) Seleção de pesquisas que comporão a amostra da análise;
3) Representação das características da pesquisa revisada;
4) Análise dos achados a partir de critérios de inclusão;
5) Interpretação dos resultados; e
6) Comunicação e publicação da revisão

A seleção das fontes, referências e palavras-chave nas publicações sobre o assunto, contido em periódicos nacionais indexados e internacionais, constitui-se no objeto desta análise, no período de 2014 a 2018, independente do método de pesquisa utilizado.

As bases de dados pesquisadas foram: Scopus; Pub Med; ProQuest; Web of Science e BVS, utilizando-se das palavras chaves: Journal Club e enfermagem ou Liderança ou gestão participativa, no período de janeiro de 2014 a dezembro de 2018, nas línguas Inglês, Francês, Português, Espanhol.

O critério para seleção dos artigos foi: incluir as categorias de artigo (original revisão de literatura, estudo de caso, multimétodo, análise de conteúdo, entre outros); artigos com resumos e textos completos disponíveis para análise no âmbito nacional e internacional.

O critério de exclusão dos artigos foi: estudos que não atendessem os critérios de inclusão mencionados, repetidos nas bases de dados os que referenciavam outras áreas da saúde que não respondiam aos objetivos. Foi realizada leitura detalhada de 97 artigos e aplicado critério de inclusão/exclusão como já mencionado.

Para coleta das informações realizou-se a organização e tabulação dos dados encontrados após leitura, sob as seguintes variáveis: Titulo, Ano, Publicação, Autores, Tipo do estudo, Resultados, Conclusão, Pais do estudo e Periódico, com enfoque no Journal Club, enfermagem baseada em evidencias, gestão participativa, ferramenta de ensino e aprendizagem e contribuições para prática, desta forma reorganizando e alimentado os dados.

A extração das informações, organização e elaboração deste banco de dados foi delineada por meio de um quadro elaborado por meio do Microsoft Word, com as seguintes variáveis: Título, Ano, Publicação, Autores, Tipo do estudo, Considerações finais e Conclusão, País do estudo e periódico. Segue abaixo o modelo do formulário: 
Quadro 1: Inserir legenda do quadro

\begin{tabular}{|l|l|l|l|l|l|}
\hline \multicolumn{6}{|c|}{ Formulário para Coleta de Dados } \\
\hline Título & Ano & Autor & Método & $\begin{array}{l}\text { Considerações } \\
\text { Finais }\end{array}$ & $\begin{array}{l}\text { Periódicos/Pais } \\
\text { publicação }\end{array}$ \\
\hline
\end{tabular}

Fonte: elaborado por (MICHELAN; SPIRI, 2019)

Posteriormente procedeu-se à análise Bibliométrica para caracterização dos estudos selecionados. Nesta fase extraímos os conceitos abordados em cada artigo selecionado, sendo comparados e agrupados por similaridade de conteúdo, sob a forma de categorias empíricas e extraídas quatro categorias tais como: Implementação da estratégia de ensino e aprendizagem Journal Club; Construção do método do Journal Club; As vantagens e desafios da implementação do Journal Club e Avaliação da estratégia Journal Club.

\section{Resultados e Discussão}

Foram identificados 97 artigos. No entanto, após avaliação e aquisição das cópias dos periódicos na integra e resumos, foram excluídos os artigos que não correspondiam aos objetivos do estudo. A amostra final foi composta por 23 artigos científicos.

Através do formulário de coleta de dados, que foi preenchido para cada artigo da amostra final do estudo (Título/ Ano/ Autor/ Método/ Considerações Finais/ Periódicos/ País de publicação).

Quadro 2: Síntese dos artigos analisados de acordo com a caracterização (título, ano, autores, metodologia, considerações finais e periódicos/ país publicação). Botucatu, 2019.

\begin{tabular}{|c|c|c|c|c|c|}
\hline Título & Ano & Autor & Método & Considerações Finais & $\begin{array}{l}\text { Periódicos/ } \\
\text { País } \\
\text { publicação }\end{array}$ \\
\hline $\begin{array}{l}\text { Fostering } \\
\text { confidence in } \\
\text { critical thinking and } \\
\text { research appraisal } \\
\text { skills } \\
\text { Journal through } \\
\text { participation: } r \text { Club } \\
\text { action an } \\
\text { study research }\end{array}$ & 2015 & WHITING D. & $\begin{array}{l}\text { Pesquisa } \\
\text { De Ação } \\
\text { Interpretativa }\end{array}$ & $\begin{array}{l}\text { O autor procurou avaliar o impacto da } \\
\text { implementação de um Journal Club em } \\
\text { um curso de enfermagem intensiva } \\
\text { como parte do primeiro ciclo de um } \\
\text { projeto de pesquisa-ação. }\end{array}$ & $\begin{array}{l}\text { Journal of } \\
\text { Pedagogical } \\
\text { Development } \\
\text { EUA }\end{array}$ \\
\hline
\end{tabular}




\begin{tabular}{|c|c|c|c|c|c|}
\hline $\begin{array}{l}\text { Nursing Journal } \\
\text { Clubs and the } \\
\text { clinical } \\
\text { nurse specialist }\end{array}$ & 2015 & $\begin{array}{l}\text { WESTLAKE } \\
\text { C. et al. }\end{array}$ & $\begin{array}{ll}\text { Estudo } & \text { de } \\
\text { Revisão } & \end{array}$ & $\begin{array}{l}\text { De acordo com o papel do especialista } \\
\text { clínico em enfermagem, o } \\
\text { desenvolvimento e implementação de } \\
\text { um Journal Club será facilitado através } \\
\text { de: formação aprimorada da equipe de } \\
\text { enfermagem; prática baseada em } \\
\text { evidências; mudanças na prática de } \\
\text { enfermagem em toda a organização e } \\
\text { investigação que pode ser conduzida } \\
\text { após a implementação do um Journal } \\
\text { Club de enfermagem. }\end{array}$ & $\begin{array}{l}\text { Clinical Nurse } \\
\text { Specialist } \\
\text { EUA }\end{array}$ \\
\hline $\begin{array}{l}\text { Journal Club for } \\
\text { prelicensure } \\
\text { nursing students }\end{array}$ & 2015 & $\begin{array}{l}\text { SCHERZER } \\
\text { R.et al. }\end{array}$ & $\begin{array}{l}\text { Relato } \\
\text { experiência }\end{array}$ & $\begin{array}{l}\text { Neste estudo os autores refletiram que a } \\
\text { medida que a ciência da enfermagem } \\
\text { continua a se expandir, os enfermeiros } \\
\text { educadores precisam prover aos alunos } \\
\text { o desenvolvimento da aprendizagem } \\
\text { sob cuidados de saúde que está em } \\
\text { constante mudança, bem como, o } \\
\text { desafio por sobrecarga de informações. } \\
\text { Eles implementaram um Journal Club } \\
\text { no programa de enfermagem, o qual foi } \\
\text { bem-sucedido em complementar o } \\
\text { ritmo acelerado de um currículo } \\
\text { acelerado. }\end{array}$ & $\begin{array}{l}\text { Nurse } \\
\text { Educator } \\
\text { EUA }\end{array}$ \\
\hline $\begin{array}{l}\text { Hospital readiness } \\
\text { for undertaking } \\
\text { evidence-based } \\
\text { practice: a survey }\end{array}$ & 2016 & $\begin{array}{l}\text { NGUYEN } \\
\text { TN, WILSON } \\
\text { A }\end{array}$ & $\begin{array}{l}\text { Estudo } \\
\text { Descritivo }\end{array}$ & $\begin{array}{l}\text { Os autores sugerem que as organizações } \\
\text { podem ser mais favoráveis à utilização } \\
\text { de pesquisa, na prática, incluindo o } \\
\text { estabelecimento de uma biblioteca, } \\
\text { Journal Club, como estratégia de } \\
\text { desenvolvimento profissional, } \\
\text { programas de orientação, envolvimento } \\
\text { dos enfermeiros na tomada de decisões } \\
\text { em nível de unidade e uma associação } \\
\text { local de enfermagem. }\end{array}$ & $\begin{array}{l}\text { Nursing and } \\
\text { Health } \\
\text { Science } \\
\text { Australia }\end{array}$ \\
\hline
\end{tabular}

Fonte: Amostra do Quadro Sinóptico dos 23 artigos selecionados (MICHELAN; SPIRI, 2019) 
Quadro 3: Distribuição das variáveis (Número de Autores, Ano de Publicação, Pais de Publicação, Método e Periódicos). Botucatu-SP, 2019

\begin{tabular}{|c|c|c|c|c|c|c|c|c|c|}
\hline \multicolumn{2}{|c|}{$\begin{array}{c}\mathbf{N}^{\mathbf{2}} \\
\text { de } \\
\text { autores }\end{array}$} & \multicolumn{2}{|c|}{$\begin{array}{c}\text { Ano } \\
\text { de } \\
\text { Publicação }\end{array}$} & \multicolumn{2}{|c|}{$\begin{array}{c}\text { País } \\
\text { de } \\
\text { Publicação }\end{array}$} & \multicolumn{2}{|l|}{ Métodos } & \multicolumn{2}{|l|}{ Periódicos } \\
\hline 1 & 4 & 2015 & 6 & EUA & 14 & Descritivo & 6 & Nurse Education Today & 4 \\
\hline 2 & 3 & 2016 & 7 & Austrália & 3 & Quantitativo & 3 & $\begin{array}{l}\text { Wordldvlews of evidence } \\
\text { basead nursing }\end{array}$ & 1 \\
\hline 3 & 3 & 2017 & 5 & Brasil & 1 & Quali-quanti & 3 & $\begin{array}{l}\text { Journal of Pedagogical } \\
\text { Development }\end{array}$ & 1 \\
\hline \multirow[t]{17}{*}{$\begin{array}{l}\text { Et } \\
\text { al. }\end{array}$} & 14 & 2018 & 5 & Canada & 1 & R.Experiência & 3 & $\begin{array}{l}\text { Revista brasileira de } \\
\text { enfermagem }\end{array}$ & 1 \\
\hline & & & & Dinamarca & 1 & Revisão & 2 & Clinical Nurse Specialist & 1 \\
\hline & & & & Finlândia & 1 & Coorte & 2 & Journal of Pediatric Nursing & 1 \\
\hline & & & & França & 1 & Estudo Caso & 1 & Rech Soins Enfermaria & 1 \\
\hline & & & & Itália & 1 & Multimetodos & 1 & Nursing Management & 1 \\
\hline & & & & & & Hibrida & 1 & $\begin{array}{l}\text { Journal of Hospital } \\
\text { Librarianship }\end{array}$ & 1 \\
\hline & & & & & & $\begin{array}{l}\text { Pesquisa } \\
\text { Ação }\end{array}$ & 1 & Applied Nursing Reserch & 1 \\
\hline & & & & & & & & Nursing Health Science & 1 \\
\hline & & & & & & & & J.Contin Educ. Nurse & 1 \\
\hline & & & & & & & & Science Direct & 1 \\
\hline & & & & & & & & $\begin{array}{l}\text { The Journal of Continuing } \\
\text { Education in Nursing }\end{array}$ & 1 \\
\hline & & & & & & & & Nurse Educator & 1 \\
\hline & & & & & & & & $\begin{array}{l}\text { Journal for Nurses in } \\
\text { Professional Development }\end{array}$ & 1 \\
\hline & & & & & & & & $\begin{array}{l}\text { Australian Journal of } \\
\text { Advanced Nursing }\end{array}$ & 1 \\
\hline & & & & & & & & $\begin{array}{l}\text { Journal of Health Care } \\
\text { Chaplaincy }\end{array}$ & 1 \\
\hline & & & & & & & & $\begin{array}{l}\text { Teaching and learning in } \\
\text { nursing }\end{array}$ & 1 \\
\hline & & & & & & & & J.Nurs Manag & 1 \\
\hline Total & 23 & & 23 & & 23 & & 23 & & 23 \\
\hline
\end{tabular}

Fonte: Amostra da distribuição das variáveis (MICHELAN; SPIRI, 2019)

Os estudos mostraram que os autores individuais são $16 \%$ dos pesquisadores, seguidos de $12 \%$ de dois a três autores que realizaram pesquisa sob o tema, bem como destacou-se os diversos autores com $60 \%$ que escreveram sob a temática. O período de publicação decorrente de 2015 foram 26,08\% das publicações internacionais, havendo um aumento de 2016 com 30,44\% de publicações, declinando em 2017/2018 com 21,74\%. Pode se dizer que 2015 houve um interesse sobre a temática, com aumento em 2016, havendo estabilidade em 2017/2018.

Os autores dos estudos inclusos utilizaram-se em sua maioria a metodologia Descritiva com 26,08\% dos artigos, seguidos de pesquisa quantitativa com $13 \%$, 
igualmente com pesquisa de relato de experiência e pesquisa quali-quantitativa com $13 \%$, pesquisas de coorte e de revisão são $9 \%$, seguidos pelos métodos qualitativos, estudo caso, estudo multimétodo, metodologias hibridas e pesquisa ação apresentaram 4,4\% dos estudos. Entretanto multimetodos foram abordados tais como: análise de conteúdo; revisão estruturada; metodologia hibrida; pesquisa ação interpretativa; qualitativa e quase experimental.

Evidentemente o interesse dos nortes americanos na estratégia de ensino e aprendizagem sob Journal Club com 60\%, bem como enfermagem baseada em evidencia, o que nos leva a pensar que se trata da devida inferência do autor do método americano, seguida dos Australianos com 12\%, bem como o interesse global pela metodologia com $4,3 \%$.

Os periódicos Nurse Education Today com 17,50\% foram os que mais publicaram sob a tematica, seguidos dos periódicos com 4,34\%, Wordldvlews of evidence basead nursing, Journal of Pedagogical Development, Revista brasileira de Enfermagem, Clinical Nurse Specialist, Journal of Pediatric Nursing, Rech Soins Enfermaria, Nursing Management, Journal of Hos pital Librarianship, Applied Nursing Reserch, Nursing Health Science, Journal Continnuing Educational Nurse, Science Direct, The Journal of Continuing Education in Nursing, Nurse Educator, Journal for Nurses in Professional Development, Australian Journal of Advanced Nursing, Journal of Health Care Chaplaincy, Teaching and learning in nursing, Journal of Nursing Management. Uma adversidade de periódicos com interesse na temática.

Após análise bibliométrica extraiu-se quatro categorias: Implementação da estratégia de ensino e aprendizagem do Journal Club; Construção do método do Journal Club; As Vantagens e os desafios da implementação Journal Club; Avaliação da estratégia Journal Club.

\subsection{Implementação da estratégia de ensino e aprendizagem do Journal Club}

De acordo com alguns artigos os mesmos retrataram o delineamento da implementação da estratégia de ensino e aprendizagem do Journal Club (JC), como parte de um ciclo pedagógico utilizando-se método da pesquisa-ação (WHITING, 2015).

Portanto, neste contexto procuraram demonstrar que $J C$ oferece um ambiente estruturado e de apoio, para adotarem e desenvolverem os princípios da enfermagem 
baseada em evidências, bem como avaliação da pesquisa e habilidades de pensamento crítico.

Corroborando com os estudos os autores refletiram que à medida que a ciência da enfermagem continua a se expandir, os enfermeiros educadores precisam prover aos alunos desenvolvimento da aprendizagem sob cuidados de saúde que está em constante mudança, bem como, desafiado por sobrecarga de informações (SCHERZER et al., 2015).

Vale ressaltar que alguns estudos apontaram que o papel do especialista clínico em enfermagem no desenvolvimento e na implementação de um $J C$ será facilitado seguindo estas diretrizes: Formação aprimorada da equipe de enfermagem, prática baseada em evidências, mudanças na prática de enfermagem em toda a organização, e investigação pode ser conduzida após a implementação de um $J C$ de enfermagem (WESTLAKE et al., 2015).

Em suma, a implementação do $J C$ que está apoiada no gestor ocorre adequadamente, pois possibilita a utilização de ferramentas como um ciclo pedagógico, fórum, on-line, determinando o momento (tempo) e implementando a padronização e a divulgação de artigos e oficinas associadas as pesquisa bibliográficas, bem como um terceiro ciclo que pode avaliar o papel dos $J C$ como um método de avaliação formativa bem como avaliação da pesquisa e das habilidades de pensamento crítico.

\subsection{Construção do método do Journal Club}

O importante papel no momento da implementação é descrever como se programa um $J C$. Sendo assim, alguns autores descrevem que no momento que se decidir e opinar por este método os participantes precisam assumir a responsabilidade por seu próprio aprendizado e pelas atividades de desenvolvimento profissional, tais como: ler e avaliar os periódicos em sua área, bem como, manter-se atualizado com tópicos gerais da pratica baseada em evidências (PURNELL; MAJID, 2017).

Portanto alguns passos devem-se ser seguidos para implementar o Journal Club tais como os propostos abaixo. (MOONAN et al., 2016; PURNELL; MAJID, 2017; WESTLAKE et al., 2015).

$>$ Decidir o $J C$ e os participantes: envolverá enfermeiros de uma determinada área ou de toda instituição?

Finalidade e objetivos 
$>\quad$ Facilitador: para mediar às discussões;

$>$ Artigo: que atenda as necessidades educacionais do publico;

$>$ Desenvolver e fornecer diretrizes para leitura dos artigos;

$>\quad$ Desenvolver um cronograma para os enfermeiros participarem da discussão;

$>$ Desenvolvimento de habilidades de avaliação crítica, a confiança na interpretação da literatura de pesquisa, a e integração da prática baseada em evidências no local de trabalho dos enfermeiros;

$>$ Auxiliar enfermeiros assistenciais a conectar-se com prática baseada em evidências, bem como avanços clínicos;

$>$ Compromisso e responsabilidade com seu aprendizado;

$>\quad$ Engajamento (tempo; frequência e disposição para aprender);

$>$ Avaliação dos resultados com base no objetivo do clube e compreensão mais profunda dos resultados do paciente.

Para elaboração do $J C$ on-line, deve-se decidir os participantes e um moderador/facilitar, no intuito de coordenar cada reunião virtual do $J C$ on-line, tendo a equipe do departamento de tecnologia de informática para determinar um formato do $J C$ on-line.

Após avaliação da equipe de TI e o desenvolvimento de um formato os autores sugerem que se tenha, por exemplo: software específico, ser acessível a todos os enfermeiros (participantes) interessados em participar, selecione um artigo que atenda as necessidades educacionais dos participantes, desenvolva e forneça diretrizes para leituras dos artigos.

Ressaltam ainda como os profissionais podem participar, e o tópico a ser discutido, desenvolver um cronograma para os enfermeiros no intuito de participar dos fóruns de discussão no JC on-line, bem como realizar avaliação dos resultados com base no objetivo do clube on-line (DOVI, 2015). O fórum virtual de discussão do JC deve promover a facilidade de uso e coordenado por alguém engajado para participar com frequência e com conhecimento (DOVI, 2015).

Consideram ainda que o uso do formato on-line, é uma ferramenta de fácil acesso, manejo educacional eficaz para enfermeiros e recomendam que os hospitais considerem os sistemas virtuais para enfermeiros integrar a prática baseada em evidências em seu fluxo de trabalho diário (PURNELL; MAJID, 2017). 


\subsection{As Vantagens e os desafios da implementação Journal Club}

A maioria dos estudos analisados observou que a literatura nos remete a vislumbrar vantagens sob o método do emprego do $J C$, para desenvolvimento profissional, seja ele na prática clínica bem como para as lideranças de enfermagem.

O JC é visto como processo de desenvolvimento de competências e de lideranças para os líderes de enfermagem, pois permite à tomada de decisão, reflexão crítica e analítica, por meio de rodas de decisão ferramentas utilizadas para promover informações para enfermeiros clínicos (FERGUSON et al., 2017; GARDNER et al., 2016; LAMAR, 2017; NGUYEN; WILSON, 2016).

Ainda, o estudo baseado em prática clínica, nos ressalta que o pragmatismo nessa prática requer uma abordagem baseada em evidências, sendo esta uma ferramenta primordial para apoiar o profissional enfermeiro, enquanto líder, assistencial e educador.

Os Journal Clubs são ferramentas de melhoria da qualidade para promover o uso da pesquisa entre profissionais de saúde, como uma estratégia com vistas para qualidade do atendimento e a segurança do paciente (MOONAN et al., 2016).

A estratégia que utiliza a via on-line, associadas a uma biblioteca de pesquisa e equipes multidisciplinar, ou seja, parceria com bibliotecários, foi a que mais apresentou resultado positivo, no que tange em engajamento e comprometimento (DRAGANOV et al., 2018).

Entretanto, alguns desafios devem ser enfrentados e aprofundados no que se refere a falta de pragmatismo e a fragilidade evidenciada entre a pesquisa e a prática (RODRIGUEZ et al., 2016).

\subsection{Avaliação da estratégia Journal Club}

A maioria dos estudos apresentaram aspectos positivos no uso do Journal Club, como uma estratégia para desenvolvimento de habilidades e competências profissionais, desde graduação a prática.

A análise crítica da literatura referente aos benefícios relatados pelo $J C$ ainda é escassa, mesmo contando com tantas vantagens positivas. Os estudos apontam que há necessidade de avaliações contínuas para estabelecer um formato e sua aplicabilidade (MCKEEVER et al., 2016). 


\section{Conclusões}

Considera-se importante a adoção do Journal Club como uma ferramenta de ensino-aprendizagem que proporciona a PBE, com a finalidade de capacitar e desenvolver, habilidades, competências e análises críticas em estudantes e profissionais. Uma estratégia promissora, porém, pouca utilizada no âmbito nacional. Embora mais conhecida internacionalmente, há necessidade de mais evidências sob sua eficácia ao longo do tempo.

Portanto, trata-se de um estudo inovador para assegurar ao profissional a possibilidade de desenvolvimento de habilidades e competências, por meio do conhecimento, tendo em vista a prática baseada em evidências.

Há muito a ser explorado, novos estudos devem ser evidenciados para que as avaliações críticas e os desafios da implementação de $J C$, possam ser superados e expandidos globalmente.

É crucial que futuros profissionais possam ter em suas mãos a garantia de uma prática livre de danos e iatrogenias, promovendo desta forma uma qualidade na assistência e a segurança do paciente.

\section{Referências}

ATALLAH, A. N.; CASTRO, A. A. Evidências para melhores decisões clínicas. São Paulo: Lemos Editorial, 1998.

DOMENICO, E. B. L. D.; IDE, C. A. C. Enfermagem baseada em evidências: princípios e aplicabilidades. Revista Latino-Americana de Enfermagem, Ribeirão Preto, v.11, n. 1, p. 115-118, jan./fev.2003.

DOVI, G. Empowering change with traditional or virtual journal clubs. Nursing, Jenkintown, v. 44, n. 10, p. 51-53, jan. 2015.

DRAGANOV, P. B. et al. Journal Club: a group of research experience. Revista Brasileira de Enfermagem, Ribeirao Preto,v.71, n. 2, p. 446-450, 2018.

FERGUSON, C. et al. The integration and evaluation of a social-media facilitated journal club to enhance the student learning experience of evidence-based practice: A case study. Nurse Education Today, Edinburgh ,v. 48,n. 1, p.123-128, 2017.

GALVÃO, C. M.; SAWADA, N. O.; TREVIZAN, M. A. Revisão sistemática: recurso que proporciona a incorporação das evidências na prática da enfermagem. Revista LatinoAmericana de Enfermagem, Ribeirão Preto, v. 12, n. 3, p. 549-556, maio./jun. 2004.

GANONG, L. H. Integrative reviews of nursing research. Research in Nursing \& Health, New York, v. 10, n. 1, p. 1-11, 1987. 
GARDNER, K. JR. et al. Implementing and Sustaining Evidence Based Practice Through a Nursing Journal Club. Applied Nursing Research, Philadelphia, v. 31, n. 1, p. 139-145, 2016.

LAMAR, R. A. Using a Virtual Journal Club for Sharing Evidence-Based Practice Recommendations in Critical Care Registered Nurses. Teaching and Learning in Nursing, Amisterdan, v. 12, n.1, p. 53-58, 2017.

LOPES, A.; LICHTENST, A. William Osler. Revista De Medicina, São Paulo, v. 86, n.3, p.185-188, jul./set. 2007.

MCKEEVER, S. et al. Creating a journal club competition improves paediatric nurses' participation and engagement. Nurse Education Today, Edinburgh, v. 37, n.1, p. 173-177. 2016.

MOONAN, M. et al. Charting the Course for a Nursing Online Journal Club: Part II. The Journal of Continuing Education in Nursing, Pitman, v.47, n. 1, p. 14-16, 2016.

NGUYEN, T. N., \& WILSON, A. . Hospital readiness for undertaking evidence-based practice: A survey. Nursing \& Health Sciences, Carlton, v.18, n. 4, p. 465-472, 2016.

PFEFFER, J.; SUTTON, R.I. Evidence-based management. Harvard Business Review, Boston, v. 84, n.1, p.62-74, 2006.

PURNELL, M.; MAJID, G. S. V. A pediatric nurses' journal club: Developing the critical appraisal skills to turn research into practice. Australian Journal of Advanced Nursing, South Melbourne, v. 34, n. 4, p. 34-41, 2017.

RODRIGUEZ, C. et al. Barriers to Participation in an Online Nursing Journal Club at a Community Teaching Hospital. The Journal of Continuing Education in Nursing, Pitman, v.47, n. 12, p. 536-542, 2016.

SCHERZER, R. et al. Journal Club for Prelicensure Nursing Students. Nurse Educator, Wakefield, Mass, v. 40, n. 5, p. 224-226, set./out. 2015.

WESTLAKE, C. et al. Nursing Journal Clubs and the Clinical Nurse Specialist. Clinical Nurse Specialist, Baltimore, v. 29, n.1, p. 1-10, jan./fev. 2015.

WHITING, D. Fostering Confidence in Critical Thinking and Research Appraisal Skills through Journal Club Participation: An Action Research Study. Journal of Pedagogyc Development, Luton, v 5, n. 2, p. 8-16, 2015.

WILSON, M. et al. Striving for evidence-based practice innovations through a hybrid model journal club: A pilot study. Nurse Education Today, Edinburgh, v. 35, n. 5, p.657-662, 2015.

Recebido em: 13 de fevereiro de 2020.

Aceito em: 22 de março de 2020. 